\title{
La financiarización de las Relaciones Laborales
}

\author{
Santos M. RUESGA \\ Universidad Autónoma de Madrid \\ ruesga@uam.es
}

Recibido: 04-01-2012

Aceptado: 26-05-2012

\section{RESUMEN}

La mundialización económica ha llevado aparejada diversos cambios estructurales de la configuración de las relaciones económica internacionales. Uno de sus efectos de mayor calado ha sido el crecimiento de la importancia del sector financiero en el conjunto de la economía hasta adquirir una centralidad insólita y que ha tenido efectos graves para el resto de relaciones económicos, una circunstancia que no ha sido exclusivamente el resultado de una mejora de la tecnología y las comunicaciones sino la consecuencia de decisiones de índole política que han transformado el entramado institucional del capitalismo a nivel mundial. Fruto de este proceso, que se ha denominado como financiarización económica, las relaciones laborales en el mundo han sufrido una serie de mutaciones de calado en los cambios en la gestión empresarial que se ha hecho más cortoplacista en todos los ámbitos, incluyendo la gestión de los recursos humanos; en el marco legal casi universal que ha reubicado el equilibrio negociador entre empresarios y asalariados para hacerlo más favorable a los primeros; y una deriva en la configuración, esencia y justificación del Estado del Bienestar en pos de una reducción de su tamaño. En conjunto, la financiarización ha supuesto en la práctica un reparto de rentas mucho más favorable hacia el capital que hacia el trabajo, nunca antes conocida en la etapa contemporánea.

Palabras clave: globalización, financiarización, relaciones laborales, crisis, gestión de empresas.

\section{Labour relations financialization}

\begin{abstract}
Economic globalisation has involved a range of structural changes that have transformed local and worldwide economic relationships. One of its most pervasive and persistent effects has been the growing importance of financial sector in the economy to the point that it has acquired a new centrality with serious consequences for the rest of economic relations - a circumstance not exclusively the result of improved technology and communications but the consequence of political decisions - that have transformed the institutional framework of global capitalism. As a result of this process, which has been called economic financialization, labour relations around the world have suffered a series of mutations due to a new corporate governance that has become more short-sighted in every areas, including human resources management; an almost universal change in legal framework both nationally and internationally that has relocated the bargaining power between employers and employees to make it more favourable to the former; and a drift in the welfare state towards a reduction of its size, configuration and essence. Overall, the financialization has meant in practice a distribution of income and power more favourable to the capital vis-a-vis labour, never known in the contemporary period.
\end{abstract}

Keywords: globalization, financialization, labour ralations, crisis, management. 


\title{
REFERENCIA NORMALIZADA
}

M. Ruesga, S. (2012). La financiarización de las relaciones laborales. Cuadernos de Relaciones Laborales Vol. 30, núm. 2, p. 409-429.

SUMARIO: Introducción. 1. Apuntes iniciales sobre el fenómeno de la globalización financiera. 2. ¿Qué se entiende por financiarización de la economía mundial?. 3. La financiarización de la economía y las relaciones económicas: visión macroeconómica. 4. La financiarización de la economía y la gestión empresarial: visión microeconómica. 5. Impacto de la financiarización de la economía en la configuración de las relaciones laborales. 6. Las consecuencias de la financiarización sobre el Estado del Bienestar. 7. Últimas reflexiones sobre las consecuencias de la financiación sobre la relación entre el capital y el trabajo. 8. Referencias bibliográficas.

\begin{abstract}
"La actual crisis económica internacional se produce debido a un crecimiento desregulado del sector financiero de la economía con una escasa supervisión, lo que, unido a otros factores económicos, ha venido generando un fuerte incremento de las desigualdades sociales a escala planetaria.

Entre otras, se puede extraer una lección del proceso de la crisis actual: los mercados son imperfectos de manera natural. Precisamente cuando la oscuridad, la falta de transparencia y la pérdida de credibilidad en la actuación de los operadores se adueñan de los mercados financieros, se ponen de manifiesto los riesgos que acarrea un tipo de crecimiento basado en la revalorización de activos financieros frente a la generación de valor en la economía real y por tanto más necesaria resulta la intervención de reguladores públicos."

(Extraído de: "El trabajo, fundamento de un crecimiento económico sostenible", declaración de 19 junio de 2009, http://www.ugt.es/fflc/actividades/09-06-19-manifiesto/090619.htm)
\end{abstract}

\section{Introducción}

El fenómeno de la globalización económica abarca un conjunto amplio de elementos y procesos socioeconómicos y políticos a tener en cuenta en el análisis en profundidad y en detalle del mismo. Aunque todos ellos se refuerzan entre sí con evidente interactividad, pueden tener causas y orígenes independientes y una correlación temporal más o menos intensa. Tal el caso de la creciente importancia del mundo de las finanzas en el ámbito de la economía mundial. Sin duda, los avances de las tecnologías de la información y la evolución de las ciencias matemáticas y actuariales, entre otros, han favorecido la expansión de un ámbito de actividad económica de principios ancestrales y arcaicos. Pero ese crecimiento inopinado no hubiese sido posible sin un entorno favorable a la expansión, sin unos marcos nacionales de regulación que han ido abandonando progresivamente las tareas de control asignadas a normas e instituciones específicas. A consecuencia de estas modificaciones en la tecnología y en el entorno institucional las transacciones financieras se han propagado en las últimas décadas a escala mundial, en tiempo real, a una velocidad y en formas desconocidas en otras épocas de la historia económica de la humanidad.

En este artículo se pretende profundizar en esos cambios acaecidos en las últimas décadas con el fin de analizar sus implicaciones en las transformaciones habidas en el mundo de las relaciones laborales. Análisis que ha de desarrollar, para llegar a un cierto grado de comprensión en la interrelación entre ambos tipos de transformaciones, financieras y laborales, en el doble plano microeconómico, el de los cambios en los modos y prevalencias en la gestión empresarial, y en el plano 
macroeconómico, donde se implican los gestores de las políticas económicas y cuyas orientaciones estimulan o retraen tales transformaciones.

\section{Apuntes iniciales sobre el fenómeno de la globalización financiera}

Aunque las relaciones económicas internacionales se mundializaron hace ya siglos, al menos a partir del momento en que Cristóbal Colón arribó en las costas de La Española a finales del siglo XV, el fenómeno que hemos convenido en denominar "globalización" (no sin ciertas dosis de imprecisión y ambigüedad respecto a lo que pretende definir) se viene aplicando a un periodo histórico del capitalismo que arranca en la década de los años sesenta del pasado siglo. Lo esencial de este proceso se representa en una fuerte expansión de la circulación de los recursos financieros y del conocimiento, como recursos productivos y del comercio de los bienes y servicios producidos a lo largo y ancho del planeta. Tal avance en la mundialización de las relaciones económicas muestra, sin embargo un discurrir asimétrico, en términos funcionales, en tanto que si bien los flujos antedichos crecen a ritmos intensos, otros flujos también de recursos productivos, como los de mano de obra, no lo hacen a tales ritmos.

Como todo fenómeno social, es susceptible de diferentes representaciones ideológicas, que se apoyan en construcciones más o menos formalizadas que denominamos teorías económicas. Así, desde el enfoque dominante en el pensamiento económico, en las últimas décadas, desde la llamada escuela neoclásica, la globalización significaría un notable avance en el desarrollo económico, apoyándose en las virtudes intrínsecas y equidistributivas que este enfoque analítico atribuya a la expansión del comercio internacional.

Otros enfoques, heterodoxos frente a las corrientes neoclásicas, hacen hincapié en los efectos asimétricos de todo orden que lleva consigo el proceso de globalización actual: asimetrías espaciales, personales, entre grupos sociales, etc., es decir, el fenómeno en cuestión arrastra consigo perdedores y ganadores en el reparto del valor añadido hipotéticamente generado por el proceso globalizador. Y si esto es así, conviene poner condiciones al proceso y no dejar su ritmo y contenidos a la decisión exclusiva de los ganadores, como ha venido ocurriendo en las últimas décadas.

$\mathrm{Y}$ es que el proceso de globalización al que asistimos se apoya en la materialización de las propuestas lanzadas desde las instituciones internacionales en pro de la apertura "in crescendo" de los espacios económicos nacionales al comercio internacional y al tráfico financiero, eliminando paulatinamente las barreras existentes a la libre circulación de bienes, servicios y dinero, que no del trabajo, como se ha señalado.

En este contexto, de apertura de las economías nacionales hacia el exterior, las posibilidades de elaborar y gestionar políticas económicas (actuaciones arbitrarias sobre el comportamiento de los agentes económicos) que impulsen el desarrollo en un espacio especifico, están fuertemente condicionadas en su contenido y dirección 
por la dinámica de la economía internacional (eufemísticamente denominado "el mercado"). El margen de libertad de las autoridades nacionales para impulsar políticas propias se reduce considerablemente en el ámbito de las relaciones económicas globalizadas. Muchos de los instrumentos tradicionales con los que operaban las políticas económicas de estabilización o de impulso al crecimiento endógeno dejan de ser operativos, al provocar su uso arbitrario efectos perversos sobre elementos básicos para el desarrollo (inversión extranjera, tasa de inflación, etc.), lo que pone de manifiesto una cierta impotencia de las políticas tradicionales (cambiarias, comerciales e incluso monetarias y fiscales) para alcanzar objetivos de equilibrio macroeconómico con crecimiento ("crecimiento económico sostenido")

Pero como señala un reconocido economista brasileño "los mercados ahora globalizados presentan nuevos defectos mucho más nocivos que los determinados clásicamente (principalmente la información imperfecta o asimétrica de los agentes y la falta de algunos mercados). Por otro lado el Estado desarrollista, proveedor de todos los beneficios se mostró en quiebra" (Bressers Pereira 1998:107). Y en este contexto ya perceptible en las últimas décadas del pasado siglo se observa que "son los flujos financieros los que experimentan un crecimiento más acelerado /.../ a expensas de las innovaciones en el mundo de la información y de la comunicación. Ello constituye una singular característica de esta fase del capitalismo que puede entenderse, por tanto, como de "globalización financiera»" (Ruesga y Heredero de Pablos 2005:17).

$\mathrm{Y}$ en este contexto evolutivo del capitalismo globalizado, conviene señalar la transformación que se produce en las instituciones de regulación económica, acompasando los cambios en la mundialización, que alteran paulatinamente el "statu quo" socioeconómico al amparo del cual había transcurrido el crecimiento económico en los países más desarrollados tras la II Guerra Mundial ("el pacto keynesiano"). Así, se observa como ya desde los años sesenta del pasado siglo se avanza una estrategia coordinada para liberalizar y desregular los mercados financieros mundiales con el objetivo explícito y deseado de insuflar eficiencia al sistema económico en su conjunto, aunque en particular al financiero, en la idea de trasladar sus potenciales beneficios al resto de actores no financieros. Pese a la inexistencia de justificación empírica expresa, acerca de los supuestos efectos positivos de esta liberalización ${ }^{1}$ (Reinhart y Rogoff 2009), tales reformas del entramado institucional se llevaron a cabo con extrema rapidez, pero los resultados han estado lejos de las expectativas: ciertamente los mercados e instituciones financieras han adquirido un papel central en el funcionamiento económico mundial, pero sometiéndolo a ciclos económicos de naturaleza mucho más intensa y prolongada que los existentes con anterioridad, con enormes pérdidas para el conjunto de los ciudadanos en caso de crisis, como se ha demostrado en el último lustro (Kose et al 2006; IILS 2009).

\footnotetext{
${ }^{1}$ Aunque sí teórica en el ámbito de los enfoques económicos ortodoxos, de las corrientes neoclásicas.
} 
En este contexto, una parte notable de la literatura académica sobre la mundialización ha puesto su atención analítica en la relación bilateral que mantienen transformaciones en los mercados financieros con el resto de subesferas del ámbito económico, en particular con las relaciones laborales (Álvarez y Medialdea 2008). Bajo la heterodoxia dentro de la ciencia económica, se ha desarrollado una rama de investigación, de presencia académica creciente, bajo la denominación común de escuela regulatoria, que en una visión más reciente y depurada y desde una noción más ecléctica en lo conceptual pero más centrada en el aparato financiero como objeto de estudio, ha ido desgranando la problemática derivada de la financiarización de la economía (Epstein 2005; Freeman 2010; Orhangazi 2008) y de la empresa (Aglietta 2000; Aglietta y Breton 2001; Dallery 2009).

Conviene pues, como paso previo al análisis del objeto de este artículo, sondear sobre el concepto de financiarización, con el fin de introducirse en las diversas interpretaciones que sobre el fenómeno en cuestión se han ido desarrollando en la literatura especializada.

\section{2. ¿Qué se entiende por financiarización de la economía mundial?}

El término "financiarización" es un neologismo aún no incorporado al lenguaje oficial español ${ }^{2}$, obviamente, como ocurre casi siempre en los últimos siglos, de origen anglosajón.

En una primera aproximación se puede entender por financiarización el "patrón de acumulación en el que los beneficios se obtienen fundamentalmente a través de los canales financieros, en lugar de a través del comercio y la producción de mercancías. Financiarización se refiere aquí a las actividades relacionadas con la disposición (o transferencia) de capital líquido a la espera de futuros de intereses, dividendos o ganancias de capital" (Krippner 2005:174-175).

$\mathrm{Y}$ el proceso de financiarización, en una expresión muy plástica se puede entender como "el creciente dominio de la industria financiera sobre el conjunto de la actividad económica, de los controladores financieros sobre la gestión de las empresas, de los activos financieros sobre el total de activos, de los valores comerciables y de las acciones entre los activos financieros y de las fluctuaciones de los mercados de valores sobre los ciclos económicos" (Dore 2000:15).

\footnotetext{
${ }^{2}$ La última edición del Diccionario de la Real Academia Española aún no lo contempla.
} 
Figura 1. Financiarización: definición y proceso
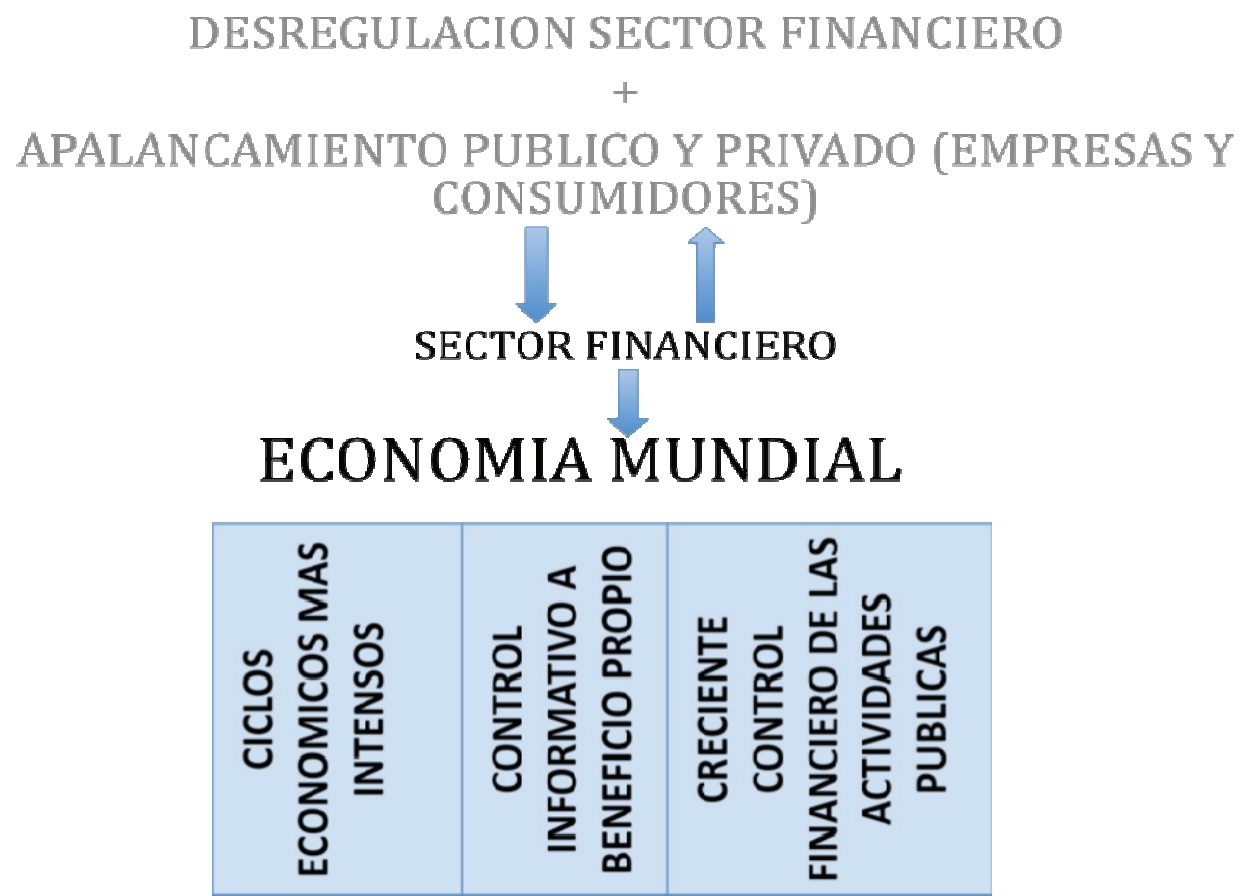

Fuente: Elaboración propia

Es decir, bajo este concepto se "alude tanto a la mayor importancia del capital financiero por oposición al capital real en lo concerniente a determinar el ritmo y el rendimiento que se espera obtener de las inversiones como a la mayor supeditación de dicha inversión a las demandas de los mercados financieros mundiales" (Ibidem). Lo que viene a significar que bajo las exigencias de los sistemas financieros las firmas del sector de manufactura y servicios se convierten en la práctica en "paquetes de bienes que se utilizan o a los que se reorienta en función de los índices de rendimiento a corto plazo que se pueden obtener." (Fligstein y Markowitz, 1990:187).

Este dominio de los actores financieros de los mercados de bienes y servicios no se constriñe al marco estrecho del mundo económico sino que expande sus tentáculos también a los ámbitos sociales y políticos, siempre enmarcado dentro de unos principios ideológicos muy concretos (Epstein 2005; Phillips 2006). En un esfuerzo constante por reinventarse, a la búsqueda de mayores beneficios a corto plazo, las empresas financieras, a través de instituciones y mercados financieros, van expandiéndose hacia otras áreas de actividad económica, política y social. Bajo los nuevos parámetros de centralidad del mundo de las finanzas, el resto de ámbitos económicos y laborales replican los grados de riesgo que tradicionalmente se asociaron 
a la esfera de las finanzas (Leyshon y Thrift 2007; Martin 2002). Lo más significativo, por tanto, es que la expansión del dominio de esta nueva "lógica financiera" deriva en la hegemonía del benefício a corto plazo como objetivo básico de la gestión empresarial imperante.

Tal reforzado rol de las finanzas se retroalimenta a partir de dos componentes: las instituciones financieras y los mercados financieros. A las instituciones financieras se les ha extendido favorablemente su marco de libertad de actuación hasta instrumentos y activos en los que con anterioridad su espacio había estado restringido por los reguladores o por otros agentes económicos ${ }^{3}$. Esta expansión de actividades se realiza con cantidades muy notables de fondos que se reproducen dentro de los mercados de capital y que se retroalimentan desde el ahorro y desde los nuevos productos financieros, implicando una ganancia de posición relativa dentro del ámbito de la economía mundial. Por su parte, los mercados financieros se perfeccionan en su sofisticación, se complican en productos y, sobre todo, se aprovechan de un nuevo marco financiero liberalizado, de tal modo que puede afirmarse que el antiguo sistema bancario ha dado paso en tan sólo unas décadas a los mercados financieros, en los que confluyen algunos de los antiguos actores, como los bancos minoristas, pero que se combinan con otros participantes con mucha mayor potencia financiera, como los fondos de pensiones, los fondos de inversión o las compañías de seguros. El monto de estos recursos pasa de estar bastante por debajo del valor de la producción industrial de los países, entendida ésta en términos del Producto Interior Bruto, a superarla holgadamente. Y frente a una opinión generalizada de que los periodos de dificultades tendrían un impacto mucho mayor sobre los activos financieros que sobre la economía real, la última crisis económica (OECD 2010).

Se atiende por tanto a una profunda transformación en la estructura funcional de los diferentes actores del capitalismo, como sistema de producción y consumo. El sector financiero alarga sus tentáculos más allá de su tradicional función de proveedor de recursos monetarios para el funcionamiento del sector productivo, es decir de "lubricante de la economía real", para asumir el papel de principal generador de resultados para el recurso capital.

Diversas interpretaciones (Álvarez y Medialdea 2009: 23) sobre el origen de este proceso de financiarización lo entienden como una reacción ante la caída la rentabilidad, en general, del recurso capital en los años posteriores al Acuerdo de Bretton

${ }^{3}$ Lo que desde los años noventa del pasado siglo se empezó a conocer de forma generaliza con el término de "ingeniería financiera", concepto que en aquel entonces tenía una lectura muy positiva en el mundo académico de la economía convencional u ortodoxa, aludiendo a la capacidad de generar novedosos instrumentos financieros que, saltando las restricciones normativas del pasado, tenían como principal característica la de generar enormes plusvalías financieras para los inversores que apostaban por productos novedosos fruto de esta "ingeniería". 
Woods. De este modo "el «corsé keynesiano» que ha mantenido durante las décadas anteriores a las finanzas como una actividad subordinadas a las necesidades de la actividad productiva, salta por los aires, mientras los mercados financieros se convierten en sí mismo en una fuente ilimitada de beneficios" (Ibidem), para imponer su lógica de acumulación al conjunto del sistema económico, en todas sus vertientes. En suma, de sistema subsidiario del tejido productivo el mundo financiero se transmuta en hegemónico, imponiendo su "lógica" en toda actividad económica.

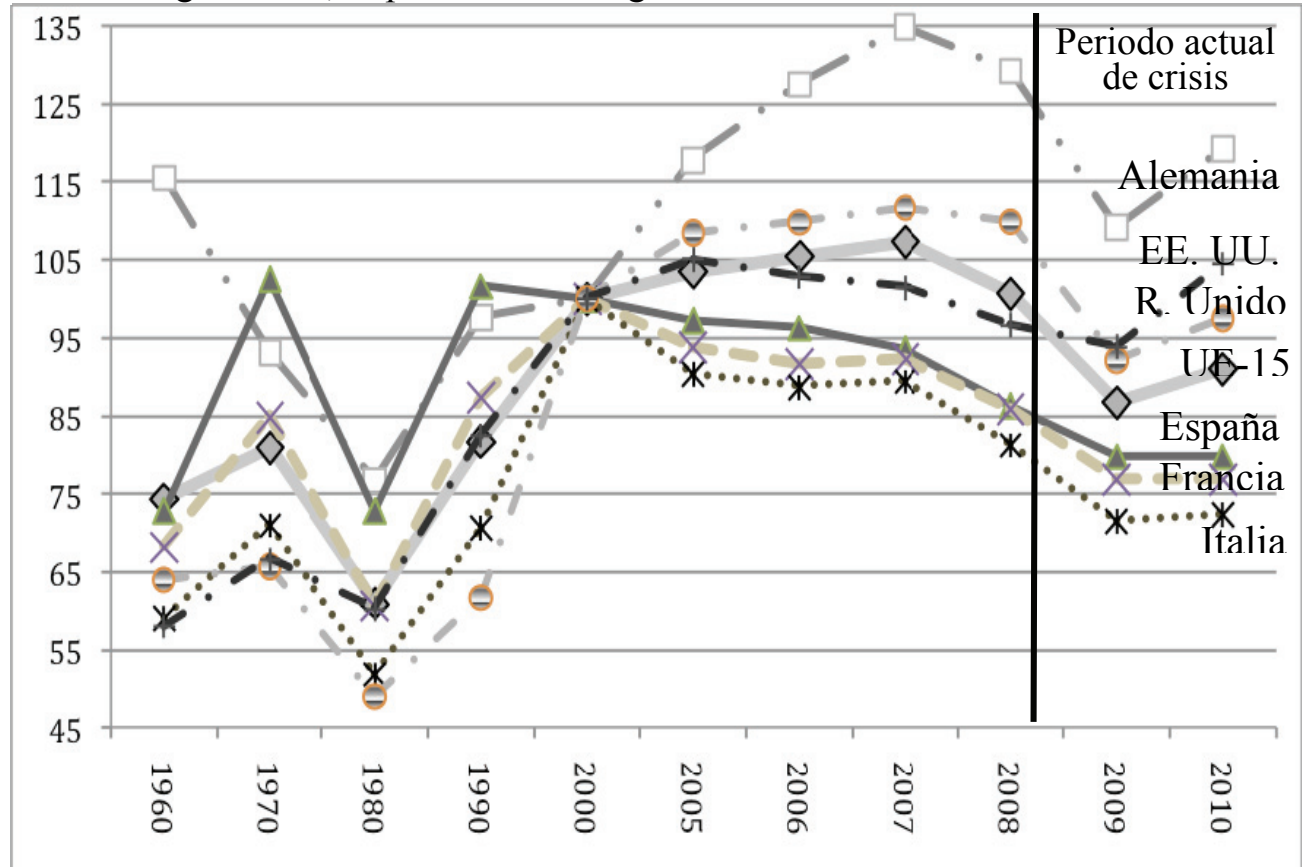

Figura 2. Evolución del rendimiento del capital (Beneficio neto/stock neto de capital) $(2000=100)$

Fuente: European Commission (AMECO (Last Update 10 November 2011)

\section{La financiarización de la economía y las relaciones económicas: visión ma croeconómica}

La financiarización de la economía tiene su plasmación e impacto más inmediatos por la vía de la propiedad de las empresas no financieras. Con grandes cantidades de liquidez e incentivos para la diversificación, el capital financiero no se ha constreñido a su tradicional mercado minorista de captación de activos y comercialización de pasivos, sino que ha pasado, en la búsqueda de incrementar la rentabilidad, a la compra y venta de empresas no financieras.

Los principales vehículos del capital financiero para la adquisición de empresas no financieras pueden dividirse en dos tipos de actores extremos: los inversores institucionales y los fondos de cobertura. Mientras los primeros buscan la rentabili- 
dad de la diversificación de una cartera con pequeñas participaciones en una multiplicidad de empresas e inversiones alternativas (Denis y McConnell 2003; Morin 2000), los hedge funds prefieren concentrar su potencial financiero y gestor en un subconjunto relativamente pequeño de empresas en el que se adquieren partes mayoritarias de la propiedad. Cualquiera que sean las formas, el resultado a efectos de la gestión de la empresa puede ser convergente.

Durante décadas los inversores institucionales se mantuvieron como el actor ideal de los gestores de las empresas: inoperantes en la gestión directa, sólo preocupados por la rentabilidad de la acción y con una iniciativa puramente dicotómica en su relación con la empresa, la de comprar y vender acciones (Roe 1994). A medida que las presiones para la rentabilidad se hicieron más acuciantes y más competitivas, los fondos institucionales en solitario o en combinación con otros inversores optaron por participar de la estrategia de los accionistas activos que, incluso con pequenas participaciones en la empresa, se sumergen en la determinación de las líneas estratégicas de la empresa.

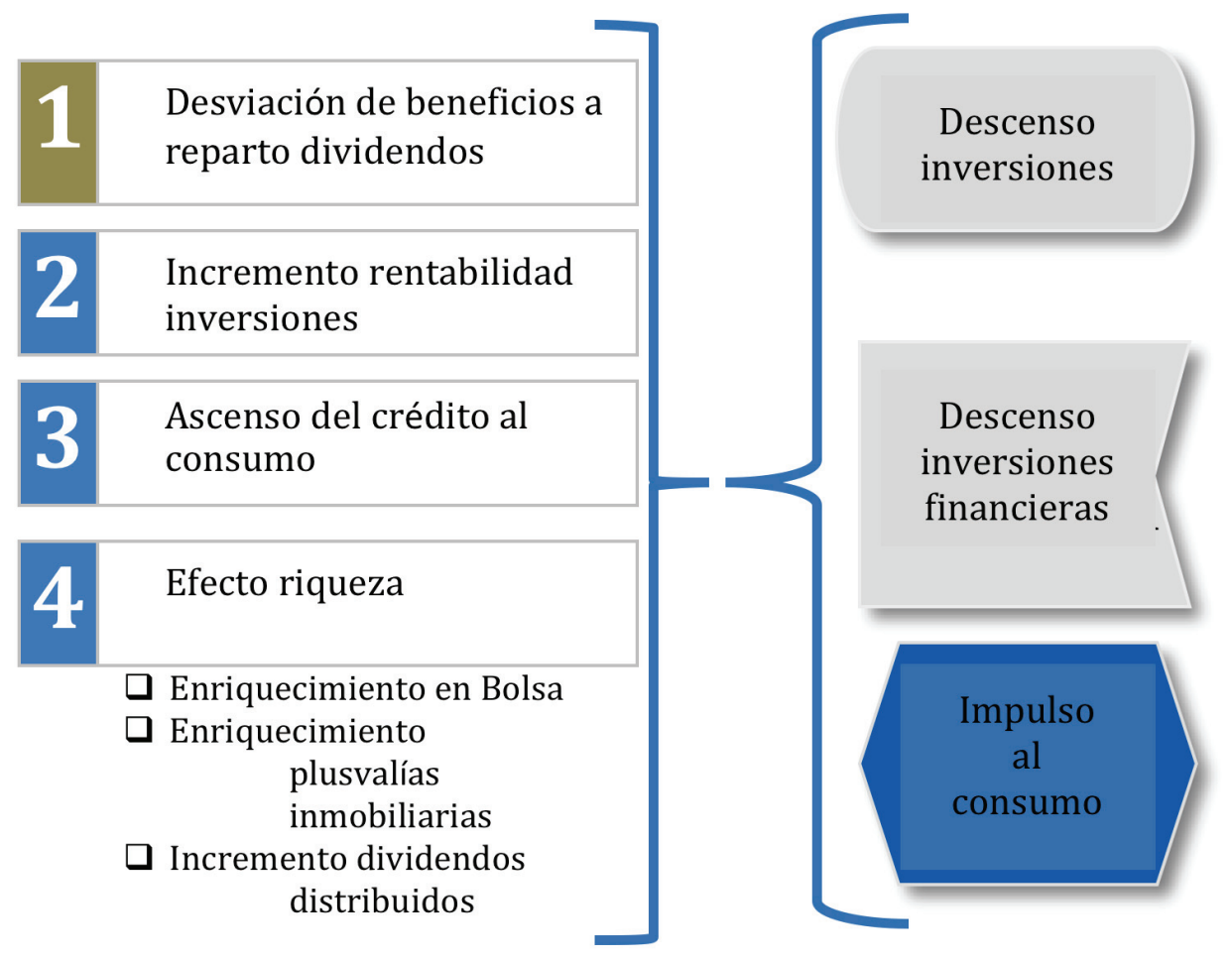

Figura 3. Financiarización: efectos macroeconómicos

Fuente: Elaboración propia 
Junto a esta delineación de la relación entre propietarios institucionales y gestores hacia formas más activas, se ha expandido el rol de los fondos de inversión cuya estrategia de beneficios pasa por la compra de la totalidad de la propiedad de empresas no financieras y la gestión directa de las mismas (Mallaby 2011), con la finalidad a corto o medio plazo de aumentar el valor del mercado de las mismas para, en última instancia, volver a vender y ejecutar plusvalías financieras.

Este análisis lleva a entender cómo la financiarización, en términos macroeconómicos, conduce a una menor tasa de inversión (Stockhammer 2005-2005 y Hein and Van Treeck) en la economía real o productiva, lo que debilita el potencial de crecimiento de la economía en el largo plazo al reducir la disponibilidad de recursos para impulsar la expansión de los componentes del capital productivo. Eso sí, en paralelo, el aumento de la rentabilidad de las inversiones financieras incrementa el atractivo de las mismas, desviando hacia ellas recursos monetarios desde otras alternativas inversoras en la economía real, con rendimientos menores y decrecientes en términos relativos, lo que autoalimenta el proceso de deterioro del potencial de desarrollo económico.

Por otro lado, algunos autores hacen hincapié en el efecto expansivo sobre el consumo de la financiarización (Boyer 2000), a causa de (figura 3), por un lado, el aumento de ingresos para el consumidor derivado del ascenso de beneficios distribuidos, de, por otro, el ascenso de la rentabilidad de las inversiones financieras, que incrementan la renta disponible de los inversores, de, en tercer lugar, la expansión del crédito al consumo (incremento del endeudamiento de los consumidores) y, finalmente, del efecto riqueza, que podría estar incrementando la propensión marginal al consumo, proveniente del ascenso en la rentabilidad de las inversiones a través del enriquecimiento en Bolsa, con las plusvalías inmobiliarias o del incremento de los dividendos distribuidos.

No obstante cabría interpretar que estos efectos sobre la expansión del consumo privado podrían estar matizados por el resultado "keynesiano" que se deriva de la contracción de la actividad productiva o el cambio en la composición capital/trabajo sobre la masa salarial de la economía.

El conjunto de estos efectos macroeconómicos pondría a determinadas economías nacionales en situaciones de desequilibrios intensos, particularmente en términos de tensiones inflacionistas, que activando políticas monetarias restrictivas (típicas en las décadas en las que el fenómeno en cuestión se desarrolla) contribuiría a frenar el crecimiento económico efectivo.

\section{La financiarización de la economía y la gestión empresarial: visión microe- conómica}

En este contexto de cambio en las relaciones entre el capital y los gestores de las empresas no financieras, el capital financiero, ya sea por la vía la propiedad, directamente, o imponiendo estrategias a los gestores, entra en una lógica de rentabilidad cortoplacista que entra en colisión sobre todo con la estrategia de acumulación y 
adquisición de tamaño que había caracterizado la gestión empresarial en los tiempos en los que la propiedad y la gestión iban unidas o bien cuando la propiedad actuaba de manera pasiva (Eatwell y Taylor 2000; Lazonick y O'Sullivan 2000; Stockhammer 2004). Es decir, o bien los gestores han renunciado a su visión tradicional de búsqueda de rendimientos a largo plazo, con una estrategia habitual de expansión de la capacidad productiva de la empresa, o bien los nuevos propietarios (los entes financieros) han sido capaces de imponer su "lógica" de recogida de valor a corto plazo. En última instancia parece como si los tradicionales problemas de confrontación entre intereses de propietarios y gestores (problemas de agencia) hubieran pasado a segundo plano, bien por connivencia ${ }^{4}$ entre ambos sujetos o bien por imposición definitiva de los intereses inmediatos de los primeros.

Esta nueva lógica de obtener rentabilidad y dividendos rápidos para competir con productos puramente financieros desde empresas no financieras tiende a provocar con mayor asiduidad políticas de recortes de gastos en el factor capital y en el factor trabajo en lugar de las más tradicionales estrategias de acumulación e inversión a largo plazo, que implicaba para el factor trabajo niveles superiores de empleo y una inversión en capital humano más intensa.

En esta perspectiva, algunos enfoques teóricos (teoría de la firma, de orientación postkeynesiana) enfatizan, a la hora de interpretar el fenómeno de la financiarización y sus efectos en la gestión empresarial, los cambios que se pueden estar produciendo entre la relación accionista-gestor. En este sentido se señala con especial énfasis como la irrupción de las entidades financieras en el entramado accionarial del sector real de la economía ha arrastrado tras de sí la preeminencia de rentabilidad a corto plazo, como fórmula de recomposición del beneficio del capital, deteriorado tras más de dos décadas de gestión al amparo del denominado pacto keynesiano.

Y en esta nueva coyuntura, la propiedad financiera, de la que los gestores serían meramente instrumentales sin capacidad o posibilidad de ejecutar objetivos propios, se embarca en todo tipo de políticas que erosionan la cantidad de trabajo contratada, ya sea por la vía de fusiones y adquisiciones que hacen redundante un parte del factor trabajo; por la externalización hacia empresas de menor tamaño y con menores salarios de partes no centrales de la actividad de la empresa; mediante deslocalizaciones productivas con los mismos objetivos; por la vía de la concentración en los segmentos y partes de cadena productiva con más beneficios en el mercado y la venta de partes colaterales a empresas de menor tamaño; por la vía directa de los despidos que ajusten en el corto plazo el tamaño óptimo de la plantilla; y por el abandono de estrategias de diversificación productiva, que solapan la actividad de

\footnotetext{
${ }^{4}$ Algo de esto puede entenderse en el comportamiento de los gestores financieros y su búsqueda de valor a corto plazo para repartir entre propiedad (dividendos) y gestión (bonus), superando así, al menos en una parte del tejido empresarial, la tradicional confrontación de objetivos entre ambos sujetos económicos, propietarios y gestores.
} 
la empresa con la diversificación propia ya existente del capital financiero. Todas estas medidas tienen un fin cortoplacista de revalorización en bolsa del capital a beneficio de los inversores y de los gestores con remuneraciones ligadas a la cotización. Pero que, en no pocas ocasiones, estas estrategias son contradictorias con la supervivencia a largo plazo de la empresa y con los niveles de empleo y producción en el corto plazo.

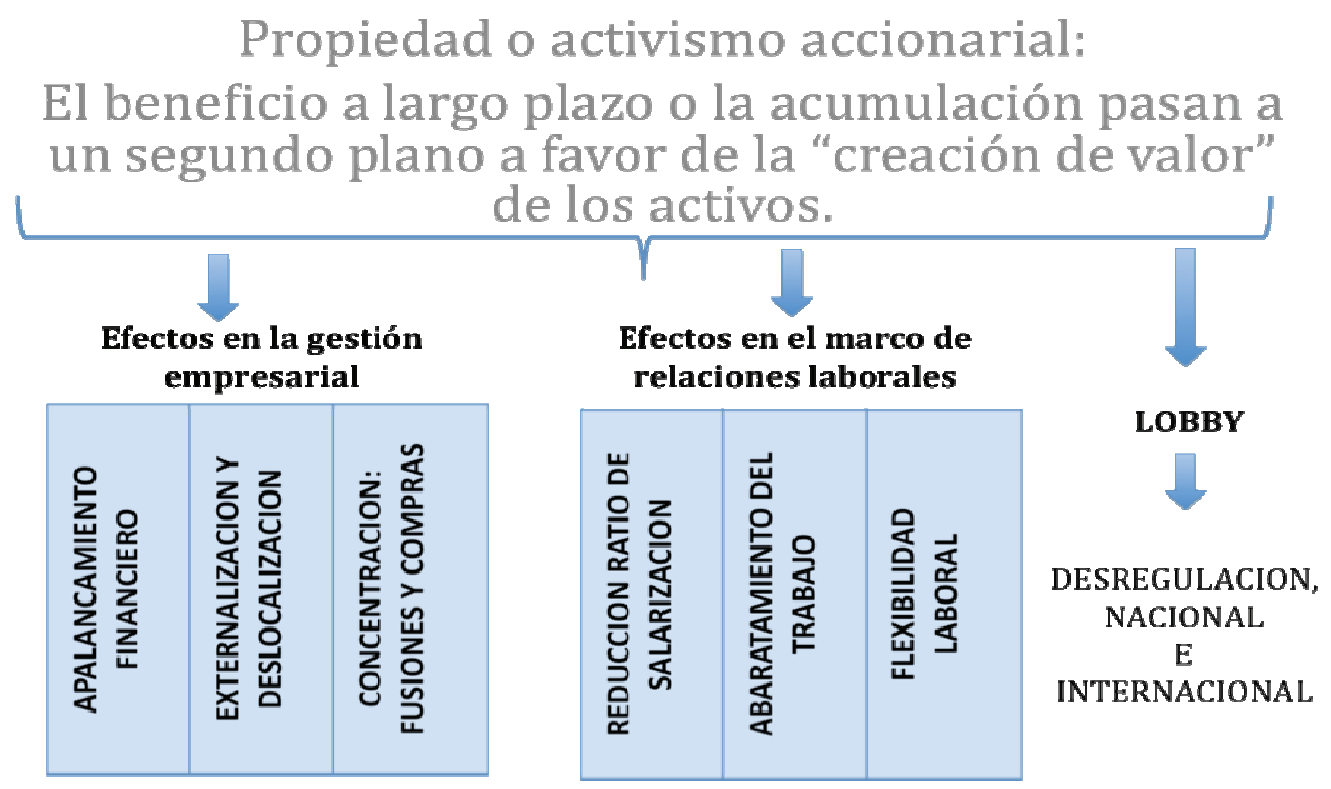

Figura 4. Financiarización: efectos microeconómicos (sobre la gestión empresarial)

Fuente: Elaboración propia

\section{Impacto de la financiarización de la economía en la configuración de las relaciones laborales}

Muchas de estas medidas, como la flexibilización laboral o la deslocalización, no podrían haber sucedido, no obstante, sin la aquiescencia o la implicación plena de los gobiernos nacionales, que ideológicamente sesgados levantaron las restricciones legales a este modelo de competencia internacional a beneficio del capital financiero y en perjuicio de los trabajadores. Además, dentro de los recursos financieros limitados de las empresas, el apalancamiento específico para comprar acciones y así retribuir más generosamente a corto plazo al accionista mediante la mera reducción del tamaño de la propiedad supone un drenaje de recursos financieros para la inversión en actividades productivas y generadoras de empleo. 
En estas nuevas estrategias corporativas se configura la presión empresarial para redefinir a la baja el papel de los sindicatos dentro de la empresa y el consecuente recorte de su poder de negociación. Por una parte, las amenazas de deslocalización y externalización de la producción y en general de reducciones en la masa salarial resultan mucho más creíbles como estrategia de negociación cuando los propietarios tienen una larga experiencia en procesos de restructuración empresarial que incorporan este tipo de estrategias al objeto de remunerar el capital financiero en el corto plazo y cuando el capital financiero no tiene nexos territoriales al centro de trabajo que puedan ser sometidos a una internalización negociadora. Por otra parte, el capitalismo financiero tiende a hacer un uso más intensivo y extensivos de instrumentos de pago financieros, como las opciones sobre acciones o las primas en función de los beneficios, en el fondo una internalización interesada de los desembolsos a los altos asalariados libre de impuestos (Domhoff 2005; Heron y Lie 2009), lo que provoca una ruptura de las estrategias comunes de negociación por parte de los trabajadores y una división general entre los objetivos de los asalariados, que ya no son exclusivamente la mejora de las condiciones salariales y laborales, porque para un cierto volumen de ellos es una parte reducida de sus ingresos.

En conjunto, se ponen en marcha estrategias con respecto al trabajo que implican una reducción del número de asalariados y un ajuste a la baja de sus salarios como la que se ha venido observando en las principales economías desarrolladas desde los años ochenta, de modo que se produce una redistribución de la renta mucho más favorable al capital, y en específico al capital financiero, que el existente en el modelo de propiedad inactiva y gestores con objetivos propios. En este ámbito, el sector público, alentado desde las instituciones internacionales y aceptado por los legisladores nacionales, ha jugado un papel de colaborador necesario y en la mayoría de los países desarrollados se han fraguado políticas que bajo la etiqueta de flexibilización generadora de empleo permitían a las empresas financieras poner en marcha sus maquinarias de gestión intensiva del capital humano, reducir los salarios y distorsionar el poder de negociación a su favor. La eficacia de estas políticas, no obstante, ha quedado en entredicho en el último lustro, cuando la propia Organización para la Cooperación y el Desarrollo Económico, que durante décadas animó las reformas laborales flexibilizadoras, llegó a la conclusión de que tras la puesta en marcha de éstas, que dañaron tan gravemente el equilibrio entre trabajo y capital, "no parece que existan razones de peso para concluir que los trabajadores en general están mejor o peor preparados para un periodo de débiles mercados de trabajo [sic] que lo que lo estaban en recesiones anteriores" (OECD 2009:40).

Las políticas flexibilizadoras en el marco de las instituciones laborales no fueron solamente el resultado de una práctica inocente y bienintencionada del proceso propio de la formación de políticas públicas sino el resultado de la convergencia de dos elementos interrelacionados. Por una parte, la relación entre los actores financieros privados y las instituciones de gobierno, en todos sus niveles, se hicieron mucho más estrechas desde principios de los años setenta, con lo que se produjo una convergencia paulatina de intereses y cultura empresarial (Johnson 2009). Por otra parte, y en parte como consecuencia de lo anterior, se produjo un cambio de los 
objetivos de la política pública: en una economía cada vez más dependiente del sector financiero, que actúa como locomotora del conjunto de la economía por la vía del apalancamiento empresarial y de la propiedad, la importancia percibida de los tipos de interés como elemento de generación de actividad económica y de la política financiera en general supera a la de los salarios, tornándose así la política monetaria y fiscal como la principal actividad pública en materia económica, muy por encima de la política industrial y laboral, que quedan subordinadas a la primera (Palley 2007; Watson 2009).

El capitalismo financiero en estrecha cooperación con las autoridades nacionales e internacionales ha redefinido el terreno de juego general de la relación entre el capital y el trabajo, provocando "que los trabajadores absorban mayores riesgos (comparados con los del periodo fordista). A través de diversos canales, los asalariados enfrentan riesgos nuevos y considerables: una creciente diferencia entre horizontes temporales (demandados por los mercados financieros) y el rendimiento productivo; requerimientos adicionales de flexibilidad laboral; [...] nuevas restructuraciones empresariales y la consiguiente segmentación laboral; y la individualización de los ingresos de los asalariados ligados a criterios de productividad y beneficios" (Álvarez y Medialdea 2008).

\section{Las consecuencias de la financiarización sobre el Estado del Bienestar}

En la redefinición a la que estamos asistiendo en la relación entre el sector público y el sector financiero, el primero parece haber renunciado a ejercer su papel de regulador económico y se lo asigna a terceros supuestamente independientes al supuesto beneficio del conjunto de los actores económicos. La intervención de parte del sector público se circunscribe a los bancos centrales o autoridades financieras creadas al efecto, que en la práctica suelen comportarse más como representantes de cara al público de la patronal del sector (Johnson 2009). En periodos de expansión y beneficios, la relación no se amplía en exceso hacia los circuitos fiscales. El sistema financiero internacional está, en todos los países, entre los que recibe un tratamiento fiscal más ventajoso bajo la falaz máxima de que es imposible gravar los capitales por su alta movilidad y que tratar favorablemente el ahorro es la mejor política para favorecer la inversión ${ }^{5}$. Este tratamiento fiscal propicio del sector financiero tiene su rúbrica cuando pasa a adquirir participaciones significativas en empresas no financieras, que por el mero cambio de propiedad pasan a participar de esas ventajas, a las que se unen la altísima cualificación del sector para minimizar

\footnotetext{
${ }^{5}$ En realidad dado que esos flujos de capitales convergen en un puñado de nodos informáticos y que las instituciones financieras reúnen cantidades ingentes de datos por su propia seguridad, su gravamen parece mucho más simple de lo que alienta la teoría con respecto a su movilidad.
} 
los pagos fiscales por la vía de la diversificación territorial, que en múltiples ocasiones suele encontrar el hecho imponible cuando ya se encuentra en un paraíso fiscal. En el mismo sentido, los procesos gerenciales que terminan con despidos de trabajadores y deslocalizaciones de partes importantes del proceso productivo suponen una merma de los ingresos fiscales del sector público al tiempo que aumentan los costes asociados al Estado del Bienestar por la vía de las prestaciones por desempleo. En definitiva, se produce la paradoja de que el proceso de financiarización de la economía real, incluso cuando muestra su cara amable, es nocivo para el mismo sector público que ha participado activamente en su liberalización.

Llegado el momento de cambio de ciclo económico, la situación del sector público con respecto al complejo financiero internacional se vuelve más compleja aún. Por una parte, el sector público en ocasiones hace frente a costosísimos rescates y a los pagos de indemnizaciones multimillonarias cuando corresponde vía los fondos de garantía que, aunque financiados por ley por las entidades financieras, se quedan muy cortos en liquidez cuando se trata de afrontar una quiebra de mediano tamaño. Por otra parte, el sector público a través de los bancos centrales se encarga de proporcionarles liquidez ilimitada, vía préstamos a bajo interés, que drenan los recursos financieros para actividades más productivas con más generación de empleo pero sin acceso directo a los bancos centrales con el argumento de que existen instituciones bancarias que tienen un tamaño que convertiría su quiebra en una catástrofe económica: too big to fail. En suma, el sector público debe cubrir buena parte de las pérdidas que deja el funcionamiento opaco a ojos de los controladores del sector financiero internacional y los escasos recursos públicos disponibles para estimular la economía se drenan por el sumidero de los mercados internacionales.

Después de décadas de políticas de libre mercado tanto en los mercados financieros como en los de trabajo, es el estado el que debe pagar la factura de la crisis en ambos, vía rescates para los primeros y por la vía de políticas laborales activas y pasivas en el segundo. El gasto en protección por desempleo se disparó en los países más sensiblemente afectados por la financiarización de la economía, desde Estados Unidos a los países bálticos pasando por España e Irlanda. Pese a que los sistemas de protección se encontraban debilitados después de años de ataques ideológicos plasmados en leyes, el coste de la factura por prestaciones por desempleo y por todos los males sociales que acarrea no fue menor. En definitiva, "el experimento del laissez faire fracasó en dos aspectos. La desregulación del sector financiero generó mayor inestabilidad en los mercados de capitales que no se compensó con un crecimiento de la producción ni de los beneficios ni de los ahorros salvo en el propio sector financiero. Mientras se desmoronaba el mundo financiero, los esfuerzos para incrementar la flexibilidad laboral sirvieron de poco para aislar a los trabajadores y a la economía real de los costes de la mala gestión financiera: costes que son, cualquiera que sea la unidad de medición, masivos" (Freeman 2010:171).

Pero los inconvenientes para el sector público de la crisis provocada desde el sector financiero no concluyeron por la vía del déficit, más o menos permanente dada la atonía de los ingresos, debido a la caída de la actividad económica y el 
aumento del gasto por las políticas contracíclicas y los estímulos keynesianos. A partir de un determinado momento, para algunos estados concretos se drenó el acceso a los mercados internacionales de deuda soberana, una enorme incongruencia con los costosos, casi permanentes y casi ilimitados rescates bancarios puestos en marcha desde los bancos centrales y desde los propios estados. El efecto a corto plazo fue una subida de los tipos de interés para la deuda soberana de algunos países que, a su vez, alimentaba las presiones del déficit público vía el pago de intereses y que se correspondía con mayores beneficios del sector financiero internacional, ése mismo que se había salvado con costosas inyecciones de capital público y que aún seguía con problemas importantes de liquidez y, en ocasiones de solvencia.

Las circunstancias empeoraron sustancialmente cuando la falta de prestamistas privados en los mercados internacionales de deuda soberana se palió con costosos planes estructurados de préstamos a altos tipos de interés, también llamados rescates, financiados por las instituciones públicas internacionales, en particular el Fondo Monetario Internacional y la Unión Europea. Estos denominados rescates llevaban adosadas unas condiciones de cambios estructurales en las economías y en los sectores públicos más propios del Consenso de Washington que se creía superado en el mundo por fracasado: flexibilidad laboral, reducción del gasto social, privatizaciones y reordenación del sistema de pensiones. En este contexto, la reducción del sector público es más que probable, como demuestra el ejemplo de la espiral griega, que conlleve una nueva contracción económica y que a su vez debilite aún más las finanzas públicas haciendo necesarios nuevos préstamos organizados.

De nuevo se produce la paradoja de que las instituciones financieras ganan en todos los contextos, ya sea en caso de expansión y en caso de recesión. Los fondos privados de pensiones, por ejemplo, durante décadas se alentaron con múltiples ventajas como complemento a un sistema público al que la evolución demográfica amenazaba con dejar en bancarrota. Estos mismos fondos se han visto beneficiados por una subida en los tipos de interés de la deuda soberana. Una de las condiciones casi universales para los préstamos de las instituciones internacionales a los estados con problemas de liquidez ha sido una reforma de los sistemas públicos de pensiones al efecto de reducir las prestaciones por jubilación en el futuro más inmediato. Es decir, el resultado de la evolución financiera deriva en unos sistemas privados de pensiones con más espacio de captación de ahorro y mejores perspectivas de rentabilidad y unos sistemas públicos mucho más ajustados, provocando por esta vía un crecimiento de las desigualdades en las sociedades más desarrolladas.

\section{7. Últimas reflexiones sobre las consecuencias de la financiación sobre la rela- ción entre el capital y el trabajo}

La alta rentabilidad ofrecida por los inversores institucionales $\mathrm{u}$ otros modelos de capital financiero organizado han provocado una creciente redefinición del patrimonio de las familias. Del modelo tradicional en el que las familias con rentas 
del trabajo acumulaban su patrimonio casi exclusivamente en bienes muebles, se ha pasado a un modelo más diversificado en el que el patrimonio financiero pasa a constituir una parte más o menos importante de su patrimonio. Esta evolución ha venido en denominarse como "capitalismo popular", aunque sea a costa de exagerar el tamaño de la propiedad en manos de las familias, su influencia real sobre las decisiones de esas empresas e ignorar los altos riesgos que corren en el proceso los asalariados (Erturk et al 2008; Wolff 2004). De hecho, lo que ha provocado el capitalismo financiero de nuevo cuño no ha sido un reforzamiento de la posición económica de los asalariados sino que se ha producido una redistribución entre grupos sociales desde la inversión empresarial, los niveles de empleo y las condiciones salariales y laborales de los trabajadores hacia los beneficios de las empresas, particularmente de las financieras, y de una parte minoritaria de sus accionistas entre los trabajadores. E incluso dentro de los trabajadores se ha producido una dualización creciente entre los que poseen más capital financiero, primero por el diferencial de crecimiento en los precios de las acciones y su rentabilidad y después en la etapa de crisis porque mantuvieron el valor de los activos financieros gracias a los rescates públicos, y los más pobres, los que más se han visto afectados por el desempleo, los que siguieron la vieja máxima de invertir en inmuebles, que sufrieron en su propio patrimonio la depreciación de los activos inmobiliarios (Gordon y Dew-Baker 2008).

Y no sólo es que el patrimonio de las familias sea más dependiente de la evolución del capital financiero en general, es que el capital financiero se encarga de asociar riesgos crecientes a los activos inmobiliarios tradicionales al insertarlas en el mercado global, es que ese mismo patrimonio inmobiliario sirve como garantía para el creciente endeudamiento de las familias (Aalbers 2008; Montgomery 2009) y es que también el intermediario retribuido entre las familias y el capital, ya sea a través de los fondos de inversión tradicionales ofrecidos por los bancos o mediante los fondos de pensiones, ha registrado un crecimiento notable al calor de políticas con un tratamiento fiscal muy favorable para las mismas. Los fondos privados de pensiones creados a partir de las rentas del trabajo han tenido una paradójica relación con los asalariados vía los mercados de capitales, dado que su participación cada vez más activa en empresas no financieras, se ha traducido en una reducción del poder de negociación del trabajo con respecto al capital, cada vez más volátil, y en una reducción general de la masa salarial relacionada con las estrategias cortoplacistas que han instaurado en las empresas.

Cabe apuntar como, en tiempos recientes parece que algún segmento de las familias asalariadas va creando un doble vínculo monetario con las empresas financieras que las hace dependientes de las rentas del capital, relegando a un plano menos unidireccional su dependencia del salario y de las rentas del trabajo en general: sus rentas actuales y futuras están cada vez menos relacionadas con su salario vía el sistema público de pensiones, que de hecho realiza actividades propias en los mercados de capitales, y con la renta salarial en general dado que las rentas del capital cada vez tienen una mayor participación en el total, en el reparto de la Renta Nacional. Esta reconfiguración del origen de las rentas de los asalariados ha 
tenido un efecto igualmente paradójico sobre la salud económica general de los países y es que la demanda agregada que mueve la economía se ha hecho menos dependiente de la masa salarial.

Por otra parte, si el patrimonio de las familias y sus decisiones de ahorro y consumo son cada vez más dependientes del capital financiero y de los precios de los mercados financieros y menos de los salarios, es previsible que disminuya la identificación de clase de las familias asalariadas (Boyer 2000; Martin 2008) y en una consecuente erosión de los logros en materia de derechos obtenidos por los asalariados desde la Segunda Guerra Mundial, que ya no se perciben como los triunfos de la inmensa mayoría sino que tienen el regusto amargo de que representan pérdidas de rentas para esa creciente combinación de asalariados y empresarios. Los antagonismos de clase que caracterizaron al periodo fordista de producción, por tanto, se diluyen y se llega a un nuevo periodo de capitalismo popular en el que las decisiones de ricos y pobres, de asalariados y rentistas, tienden a converger y la concordancia entre la empresa y sus trabajadores, propia del análisis industrial de las relaciones laborales, se aminora puesto que entre estos últimos se adquiere un cierto grado de independencia en materia de rentas, más ficticia que real puesto que viaja vía los fondos de inversión y los mercados financieros hasta el núcleo de la propiedad de las empresas.

\section{Referencias bibliográficas}

Aalbers, M.B. (2008). The financialization of home and the mortgage market crisis. Competition and Change, 12:148-166.

Aglietta, M. (2000). Shareholder value and corporate governance: some tricky questions. Economy and Society, 29:146-59.

Aglietta, M. y R. Breton. (2001). Financial systems, corporate control and capital accumulation. Economy and Society, 30:433-66.

Álvarez, N. y B. Medialdea. (2008). Financial globalization and labor: employee shareholding or labor regression? Amherst: Political Economy Research Institute.

Álvarez, N. y B. Medialdea. (2009). Financiarizacion, crisis económica y socialización de pérdidas, Viento del Sur, número 100, enero:21-32

Boyer, R. (2000). Is a finance-led growth regime a viable alternative to Fordism? A preliminary analysis. Economy and Society, 29:111-45.

Dallery, T. (2009). Post-Keynesian theories of the firm under financialization. Review of Radical Political Economics, 41:492-515. 
Denis, D.K. y J.J. McConnell. (2003). International corporate governance. Journal of Financial and Quantitative Analysis, 38:1-36.

Domhoff, G.W. (2005). Wealth, income, and power. Santa Cruz: University of California.

Dore, R. (2000). Stock market capitalism: welfare capitalism: Japan and Germany vs the Anglo-Saxons. Oxford: Oxford University Press.

Eatwell, J. y L. Taylor. (2000). Global finance at risk. Cambridge: Polity.

Epstein, G.A., ed.(2005). Financialization and the world economy. Northampton: Edward Elgar.

Erturk, I., J. Froud, S. Johal, A. Leaver y K. Williams. (2007). The democratization of finance? Promises, outcomes and conditions. Review of International Political Economy, 14:553-75.

Fligstein N. y L. Markowitz. (1993). Financial Reorganization of American Corporations in the 1980s, en W. J. Wilson (ed), Sociology and the Public Agenda. Newbury Park: Sage:185-206.

Freeman, R.B. 2010. It's financialization! International Labour Review, 149:163-83.

Gordon, R.J. y I. Dew-Baker. (2008). Controversies about the rise of American inequality. Cambridge: National Bureau of Economic Research.

Heron, R.A. y E. Lie. (2009). What fraction of stock options grants to top executives have been backdated or manipulated? Management Science, 55:513-25.

Hein, E., and T. van Treeck. (2007). Financialisation in Kaleckian/post-Kaleckian models of distribution and growth. IMK Working Paper $\mathrm{n}^{\circ}$ 7/2007.

IILS. (2009). World of work: the global jobs crisis and beyond. Ginebra: International Institute of Labour Studies.

Johnson, S. (2009). The quiet coup. Atlantic Monthly, mayo.

Kose, M.A., E. Prasad, K. Rogoff y S.J. Wei. (2006). Financial globalization: a reappraisal. Washington: International Monetary Fund.

Lazonick, W. y M. O'Sullivan. (2000). Maximizing shareholder value: a new ideology for corporate governance. Economy and Society, 29:13-35. 
Leyshon, A. y N. Thrift. (2007). The capitalization of almost everything: the future of finance and capitalism. Theory, Culture and Society, 24:97-115.

Mallaby, S. (2010). More money than God: hedge funds and the making of a new elite. Nueva York: Penguin.

Martin, R. (2002). Financialization of daily life. Filadelfia: Temple University Press.

Montgomery, J. (2009). The pursuit of (past) happiness? Middle-class indebtedness and American financialisation. New Political Economy, 14:1-24.

Morin, F. (2000). A transformation in the French model of shareholding and management. Economy and Society, 29:36-53.

OECD. (2009). Employment outlook. París: Organisation for Economic Cooperation and Development.

OECD. (2010). Annual statistics on institutional investors' assets. París: Organisation for Economic Cooperation and Development.

Orhangazi, Ö. (2008). Financialization and the U.S. economy. Cheltenham: Edward Elgar.

Palley, T.I. (2007). Financialization: what it is and why it matters. Annandale-onHudson: Levy Institute.

Phillips, K. (2006). American theocracy: the peril and politics of radical religion, oil, and borrowed money in the $21^{\text {st }}$ century. Nueva York: Penguin.

Reinhart, C. y K. Rogoff. (2009). Eight centuries of financial folly. Princeton: Princeton University Press.

Roe, M. (1994). Strong managers, weak owners: the political roots of American corporate finances. Princeton: Princeton University Press.

Rossman P. (2006). Financiarización: Nuevas Sendas hacia las Ganancias, Nuevos Desafíos para los Sindicatos, Educaciõn Obrera, la revista de la Oficina de Actividades para los Trabajadores (ACTRAV) de la OIT, 1/2006, No. 142.

Ruesga, S.M. y Heredero de Pablos, M.I. (2005). De discriminaciones laborales y ajustes salariales en el entorno globalizador, Revista de Economía Mundial, 12:15-24.

Stockhammer, E. (2004). Financialization and the slowdown of accumulation. Cambridge Journal of Economics, 28:719-41. 
Stockhammer, E. (2005-6). Shareholder value orientation and the investment profit puzle. Journal of Post Keynesian Economics, 28 (2): 193-215.

Watson, M. (009). Planning for a future of asset-based Welfare? New Labour, financialized economic agency and the housing market. Planning Practice, 24:41-56.

Wolff, E.N. (2004). Recent trends in living standard in the United States. En What has happened to the quality of life in the advanced industrialized nations?, editado por E.N. Wolff. Cheltenham: Edward Elgar. 